ANÁLISIS CUALITATIVO DE LA PERCEPCIÓN DEL MARKETING COMO FACTOR COMPETITIVO INTERNO: CASO DE UNA MICROEMPRESA FERRETERA DE YUCATÁN

\title{
ANÁLISIS CUALITATIVO DE LA PERCEPCIÓN DEL MARKETING COMO FACTOR COMPETITIVO INTERNO: CASO DE UNA MICROEMPRESA FERRETERA DE YUCATÁN
}

López Arroyo María de los Ángeles*, Morales González María Antonia**, Madero Llanes

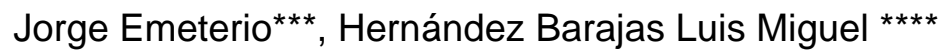

*Maestra en Planificación de Empresas y Desarrollo Regional. Departamento de Ingeniería Industrial. Instituto Tecnológico de Mérida.

${ }^{*}$ Doctor (a) en Ciencias en Planificación de Empresas y Desarrollo Regional. Departamento de Ingeniería Industrial. Profesor (a) Investigador (a). Instituto Tecnológico de Mérida.

***Doctor en Educación. Departamento de Ingeniería Industrial. Profesor Investigador. Instituto Tecnológico de Tapachula.

Dirección para recibir correspondencia: angeles21_88@hotmail.com 
ANÁLISIS CUALITATIVO DE LA PERCEPCIÓN DEL MARKETING COMO FACTOR COMPETITIVO INTERNO: CASO DE UNA MICROEMPRESA FERRETERA DE YUCATÁN

\section{RESUMEN}

Objetivo: Conocer la percepción de una microempresa del subsector ferretero de Mérida, Yucatán en cuanto a cómo aplica las herramientas operativas del marketing (producto, precio, plaza y promoción).

Material y método: La investigación fue de enfoque cualitativo, la información se obtuvo mediante entrevistas estructuradas. Los resultados se basan en un análisis de contenido de nivel sintáctico realizado en el software Nvivo 10.0.

Resultados: En la herramienta enfocada al producto las percepciones evidenciaron la ausencia de una metodología para determinar los productos que se comercializan, de igual forma en las herramientas enfocadas al precio y plaza se observó la ausencia de una metodología para la fijación de precios y la ubicación de la empresa respectivamente y finalmente herramienta publicidad, se observó divergencia en su objetivo principal el cual recae en la promoción de los productos o servicios y no en posicionar el nombre de la empresa como fue el caso de la presente investigación.

Conclusiones: La microempresa estudiada realiza las actividades que implican las herramientas operativas del marketing, sin embargo, se observó que las herramientas producto, precio y plaza se desarrollan empíricamente.

Palabras clave: Competitividad. Marketing. Ferreterías. Percepciones. Nvivo 10.0. 


\section{ABSTRACT}

Objective: To know the perception of a microenterprise subsector Ironmonger Merida, Yucatan on how to apply the operational tools of marketing (product, price, place and promotion).

Material and method: The research was qualitative approach; information was obtained through structured interviews. The results are based on a content analysis performed on the syntactic level Nvivo 10.0 software.

Results: Focused on product perceptions tool showed the absence of a methodology to determine which products are marketed, just as the tools focused on price and place the absence of a methodology for pricing and location are observed respectively and advertising company eventually was observed divergence tool in its primary objective which lies in the promotion of products or services and not to position the company name as was the case in this investigation.

Conclusions: Microenterprises studied performs the operational activities involving marketing tools, however, noted that the product, price and square tools are developed empirically.

Key words: Competitiveness. Marketing. Hardware. Perceptions. Nvivo 10.0. 
De acuerdo al Instituto Nacional de Estadística y Geografía (INEGI), el subsector comercio al por menor de productos de ferretería, tlapalería y vidrios representa una de las principales potencialidades económicas del país; y en el estado de Yucatán el panorama no es diferente, dicho subsector cuenta con 1288 puntos de venta (ferreterías), en dicho estado, las cuales generan aproximadamente 5994 empleos; de ellos el 56.31\% son generados por microempresas, $16.88 \%$ por pequeñas empresas, $21.07 \%$ por medianas empresas y únicamente el $5.74 \%$ por grandes empresas; las cifras anteriores lo ubican dentro de los 15 subsectores más importantes económicamente para la región (INEGI, 2012).

Sin embargo, Castillo (2015), afirma que a nivel nacional el subsector comercio al por menor de productos de ferretería, tlapalería y vidrios atraviesa una situación difícil debido a que en los últimos años su desempeño ha ido en descenso, lo cual se ve reflejado en su índice de ventas realizado por el INEGI, dicho índice registró en el 2013 una caída de $4.32 \%$ respecto al 2012 y para el periodo de enero-agosto de 2014, el descenso fue de 5.4\% en relación al mismo periodo de 2013. De igual forma, en Yucatán, en 2013 el INEGI explicó que el descenso de 2.2\% anual que registraron las ventas al menudeo en el Estado se debió a las reducciones en los subsectores de artículos de ferretería, tlapalería y vidrios, entre otros (INEGI, 2013); mientras que para febrero de 2014 el descenso fue de $1.7 \%$ respecto a febrero de 2013 , derivado nuevamente de las reducciones en diversos subsectores, entre ellos el de artículos de ferretería, tlapalería y vidrios, resaltando como las que presentan disminución a Mérida, Yucatán (INEGI, 2014).

Al respecto, Carmona (2015), señala que en los últimos 3 años la presencia de Home Depot en México ha sepultado a cientos de negocios familiares de ferreterías o tlapalerías que se han visto obligadas a cerrar ante su imposibilidad de competir con la cadena. Sumado a lo anterior, Home Depot informó que busca ampliar su presencia en México para el 2016, mediante tiendas de formato pequeño, mismas que han resultado un éxito para la cadena en la prueba piloto efectuada en Querétaro (Home Depot, 2015), lo cual podría significar la desaparición de más ferreterías y tlapalerías familiares a nivel nacional. 
Por lo anterior, es evidente que las microempresas ferreteras requieren de estrategias que garanticen su permanencia en el mercado; al respecto, diversos autores afirman que el marketing es un factor esencial para la competitividad de las empresas (Aguirre y Aparicio, 2002; Castillo, Bojórquez y Pérez, 2013; Rubio y Aragón, 2002), y que el declive de éstas se debe a un marketing no rentable (O'Shaughnessy, 1991). Desafortunadamente las microempresas se caracterizan principalmente por tener desventaja sobre las grandes corporaciones en el proceso de realización de marketing (Paredes 2011). Lo anterior, debido a la falta de conocimiento de la aplicación eficiente de dicho proceso (Stokes, 2000), y a lo costoso e inaccesible que les resulta realizar una publicidad en grandes dimensiones como el de las compañías transnacionales (Bacon, 1996).

No obstante, Paredes (2011), sostiene que sin importar el tamaño de las empresas, el marketing es fundamental para garantizar su competitividad, principalmente se requiere de la gestión eficiente de sus herramientas operativas, las cuales se conocen como marketing mix y se consideran el instrumento básico y clave para la implementación eficaz del marketing (Soriano, 1990). Por ello, debido a la problemática anteriormente mencionada, aunado al desconocimiento de la forma en que se efectúa el marketing en las microempresas ferreteras de Mérida y tomando como referencia a Caro y Díez (2005), quienes afirman que el enfoque cualitativo se ha utilizado para comprender a profundidad el funcionamiento de las empresas; ésta investigación se centró en el análisis de un estudio de caso de enfoque cualitativo aplicado a una microempresa del subsector ferretero de Mérida, Yucatán. El objetivo fue conocer su percepción en cuanto a cómo aplica las herramientas operativas del marketing.

\section{MARCO TEÓRICO}

Diversos autores coinciden en que el marketing consiste en desarrollar de forma eficiente un conjunto de actividades para satisfacer las necesidades de los consumidores mediante un producto o servicio (Kotler y Keller, 2006; López, Mas y Viscarri, 2008), dichas actividades se deben desarrollar mediante un adecuado proceso de planificación y 
ejecución con la finalidad de identificar, crear, desarrollar y servir a la demanda (Arriaga, Avalos y De la torre, 2012). El propósito de la mercadotecnia es satisfacer a los clientes mediante la realización de actividades con la mayor eficiencia, lo cual significa obtener óptimos resultados con el mínimo esfuerzo, tiempo y dinero mediante el uso de diferentes técnicas como la promoción de ventas, investigación de mercados, etcétera (Mercado, 2004).

La mercadotecnia está integrada por variables controlables y variables no controlables; las primeras son aquellas sobre las que la empresa puede ejercer dominio y control y se conforman por la denominada mezcla de mercadotecnia; y las segundas se conforman por aquellos factores sobre los que no puede actuar, como lo son los aspectos políticos y legales, aspectos socioculturales, competencia, aspectos económicos y aspectos demográficos pero que sin lugar a dudas todas las empresas deben poner especial atención por los efectos que pueda o no causar en su estrategia de marketing (Bustamante, 2001).

Por ello, esta investigación se centra en la mezcla de mercadotecnia la cual se define como el "conjunto de herramientas controlables e interrelacionadas de que disponen los responsables de marketing para satisfacer las necesidades del mercado y, a la vez, conseguir los objetivos de la organización" (Rodriguez et al., 2006 p.69).

La mezcla de mercadotecnia se integra por las cuatro herramientas operativas del marketing, estas se conocen como las "cuatro P"; es decir, el producto, el precio, la plaza y la promoción; para que estas herramientas sean eficaces, las empresas deben partir de una análisis de su situación actual considerando sus recursos y capacidades y en base a ello diseñar su estrategia de marketing considerando las tendencias en el mercado (ver figura 1) (Dvoskin, 2004). 


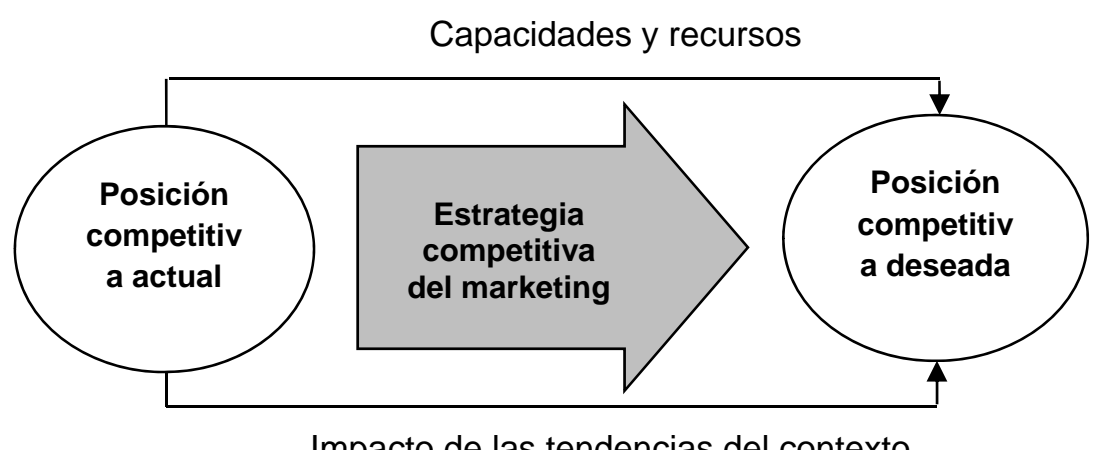

Impacto de las tendencias del contexto

Figura 1. Interrelación entre el marketing y competitividad.

Fuente: Dvoskin (2004).

Arriaga et al., (2012), definen estas cuatro herramientas operativas, también conocidas como marketing mix o mezcla de mercadotecnia:

- El producto: Este puede ser palpable o impalpable e implica entre otras cosas determinar los productos a comercializar y contar con amplitud en el surtido de productos con el objetivo de cumplir las necesidades de sus clientes.

- El precio: Es el valor económico que se debe pagar por un bien o servicio y el cual debe considerar un margen mínimo de costo hacia el cliente con el objetivo de evitar pérdidas cuando la empresa realiza promociones; considerando de igual forma los precios de la competencia.

- La plaza: Consiste en determinar cuáles serán las sucursales, almacenes minoristas, etcétera ya que estos constituyen el medio por el cual se distribuirán los productos o servicios de la empresa.

- La promoción: Son actividades realizadas por la empresa que tienen como principal objetivo posicionar un producto o servicio para motivar la compra en los consumidores o usuarios mediante la emisión de mensajes por medio de folletos, radio, televisión, exposiciones. 
Kotler y Armstrong (2008), coinciden con las definiciones anteriores; sin embargo, en la herramienta enfocada a la plaza acentúan la importancia de seleccionar una ubicación idónea que permita a las empresas estar cerca de sus clientes potenciales.

\section{OBJETIVOS DE LA INVESTIGACIÓN}

Mediante el presente estudio se pretendió cumplir con el siguiente objetivo:

- Conocer la percepción del marketing de una microempresa del subsector comercio al por menor de productos de ferretería, tlapalería y vidrios de la Ciudad de Mérida, Yucatán, en cuanto a cómo aplica las herramientas operativas del marketing (producto, precio, plaza y promoción).

Tomando como referencia lo anterior, con el objetivo de conocer si la empresa en estudio emplea un procedimiento para determinar su cartera de productos, se buscó conocer la percepción de ésta sobre el surtido de productos que actualmente posee; se analizaron las consideraciones para el establecimiento de precio a sus productos; en la herramienta enfocada a la plaza, se investigaron las consideraciones para determinar la ubicación de la empresa y finalmente en la herramienta promoción, se cuestionó a la empresa las razones por las que realiza o no realiza publicidad.

\section{MATERIAL Y MÉTODO}

La presente investigación se centró en el análisis de un estudio de caso de tipo intrínseco ya que se buscó tener una mejor comprensión sobre la forma en que una microempresa aplica las herramientas operativas del marketing, mas no se pretendió generar ninguna teoría ni generalizar los resultados (Stake, 2007).

Enfoque: El estudio fue de enfoque cualitativo debido a que el análisis se realizó a partir de las palabras propias de las personas consideradas en la investigación (Taylor y Bogdan, 1986 citado en Rodríguez, Gil y García, 1999). 
Unidad de análisis: El sujeto de estudio corresponde a una microempresa con 18 años de antigüedad que se integra por el propietario y 3 empleados, dicha empresa pertenece al subsector 467: Comercio al por menor de artículos de ferreterías, tlapalerías y vidrios de acuerdo a la codificación del Sistema de Clasificación Industrial de América del Norte (SCIAN, 2007), y se encuentra registrada bajo el régimen de actividad física empresarial en la ciudad de Mérida, Yucatán.

Herramienta de recolección de información: Para obtener la información se emplearon entrevistas ya que estas se consideran uno de los principales métodos utilizados en las investigaciones cualitativas (Hernández, Fernández y Baptista, 2010). Las entrevistas fueron de tipo estructuradas, las cuales, de acuerdo a Cerda (1998, citado en Bernal, 2010), se realizan a partir de un formato de aspectos a investigar previamente diseñado; en el presente estudio las entrevistas se elaboraron con preguntas referentes a cada una de las herramientas operativas del marketing, es decir, producto, precio, plaza y promoción.

Posteriormente, se aplicaron al empresario y a los 3 empleados de la empresa, esto con el objetivo de tener una información más integral y comprender a profundidad como se desarrollan dichas herramientas operativas.

Procedimiento de análisis de información: El procesamiento de datos se efectuó en el software Nvivo versión 10.0, ya que este figura como apoyo en el tratamiento de datos cualitativos (Hernández et al. 2010), y ha sido utilizado para conocer percepciones de entrevistados (Camacho y Pérez, 2014). Con el cual se realizó un análisis de contenido de nivel sintáctico ya que se interpretaron las frases y/o expresiones que contenían la palabras más frecuentes (Gil, Conti, Pinzon, Prieto, Solas, \& Cruz, 2002).

Para ello, una vez obtenidas las respuestas se introdujeron en el software mencionado y se obtuvieron en cada una de las respuestas las 20 palabras más frecuentes con una longitud mínima 5 letras (con el objetivo de eliminar palabras gramaticales carentes de valor analítico como artículos, preposiciones, muletillas, etcétera y únicamente obtener palabras léxicas para ser interpretadas), de las cuales se eligió la más frecuente y a partir 
ANÁLISIS CUALITATIVO DE LA PERCEPCIÓN DEL MARKETING COMO FACTOR COMPETITIVO INTERNO: CASO DE UNA MICROEMPRESA FERRETERA DE YUCATÁN

de ésta se obtuvo una red léxica, la cual permitió visualizar e interpretar expresiones ligadas a dicha palabra y en base a ello conjeturar las percepciones del empresario y los empleados.

\section{RESULTADOS}

A continuación, se presentan los resultados obtenidos en cada una de las herramientas operativas del marketing tras el análisis de las respuestas obtenidas en las cuatro entrevistas mediante el software Nvivo 10.0.

La tabla 1, muestra las 20 palabras más frecuentes utilizadas durante las entrevistas por el propietario y los empleados de la empresa al cuestionar sobre los aspectos relacionados al producto, precio, plaza y promoción; como se mencionó anteriormente se obtuvieron las palabras con un mínimo de 5 letras para evitar las palabras carentes de valor analítico. 
ANÁLISIS CUALITATIVO DE LA PERCEPCIÓN DEL MARKETING COMO FACTOR COMPETITIVO INTERNO: CASO DE UNA MICROEMPRESA FERRETERA DE YUCATÁN

Tabla 1

Frecuencia de palabras en herramientas operativas del marketing

\begin{tabular}{|c|c|c|c|}
\hline $\begin{array}{l}\text { Herramientas } \\
\text { operativas del } \\
\text { marketing }\end{array}$ & Palabras & Frecuencia & $\begin{array}{l}\text { Porcentaje } \\
\text { ponderado } \\
(\%) \\
\end{array}$ \\
\hline \multirow{7}{*}{ Producto } & Siempre & 10 & 2.39 \\
\hline & Productos & 7 & 1.67 \\
\hline & Cuando, porque & 6 & 1.43 \\
\hline & Clientes & 5 & 1.19 \\
\hline & Tienen, trato & 4 & 0.95 \\
\hline & $\begin{array}{l}\text { Considero, ejemplo, empresa, lámparas, } \\
\text { vienen }\end{array}$ & 3 & 0.72 \\
\hline & $\begin{array}{l}\text { Ahora, cantidades, comprar, diferentes, } \\
\text { herramientas, marcas, momento, muchas }\end{array}$ & 2 & 0.48 \\
\hline \multirow{6}{*}{ Precio } & Margen & 10 & 2.75 \\
\hline & Precio & 7 & 1.93 \\
\hline & Utilidad & 6 & 1.65 \\
\hline & Productos & 5 & 1.38 \\
\hline & Generalmente & 4 & 1.1 \\
\hline & $\begin{array}{l}\text { Descuento, dueño, ellos, empresas, escrita, } \\
\text { establece, gastos, gente, hasta, limpieza, } \\
\text { maneja, muchas, notas, podría proveedor }\end{array}$ & 2 & 0.55 \\
\hline \multirow{4}{*}{ Plaza } & Cerca & 8 & 2.65 \\
\hline & Clientes, fraccionamiento & 4 & 1.32 \\
\hline & $\begin{array}{l}\text { Cuando, empresa, lugar, mayoría, nuestros, } \\
\text { permite, porque, proveedores }\end{array}$ & 3 & 0.99 \\
\hline & $\begin{array}{l}\text { Calle, ciudad, considero, estamos, estar, } \\
\text { estuviera, importante, negocios, personas }\end{array}$ & 2 & 0.66 \\
\hline \multirow{5}{*}{ Promoción } & Empresa & 15 & 4.49 \\
\hline & Conocer, importante & 5 & 1.5 \\
\hline & Ayuda, productos, publicidad & 4 & 1.2 \\
\hline & Personas, servicios & 3 & 0.9 \\
\hline & $\begin{array}{l}\text { Ejemplo, enfrente, existe, fanpage, } \\
\text { fraccionamiento, pueden, hemos, muestra, } \\
\text { tlapalería, venden, abrimos, anuncié }\end{array}$ & 2 & 0.6 \\
\hline
\end{tabular}

Fuente: Elaboración propia en base a información arrojada por el software Nvivo 10.0.

A continuación, se muestra el análisis sintáctico por medio de redes léxicas derivadas de la palabra más frecuente en cada una de las herramientas operativas del marketing. 
Al analizar la parte de la herramienta enfocada al producto, se encontró que el adverbio de tiempo "siempre" con 10 referencias y un $2.39 \%$ ponderado del total en las 4 entrevistas, fue la palabra más frecuente ante la pregunta sobre cómo consideran el surtido de la empresa.

La red léxica de dicha palabra se observa en la figura 2, de la cual se extraen las frases "Por el momento sí, porque siempre me ha permitido tener una gran rotación..." y “...siempre que vienen los clientes a la tienda siempre se tiene lo que desean...”, de ellas se deduce que los empleados y el propietario de la empresa atribuyen un surtido óptimo a la rotación de productos...

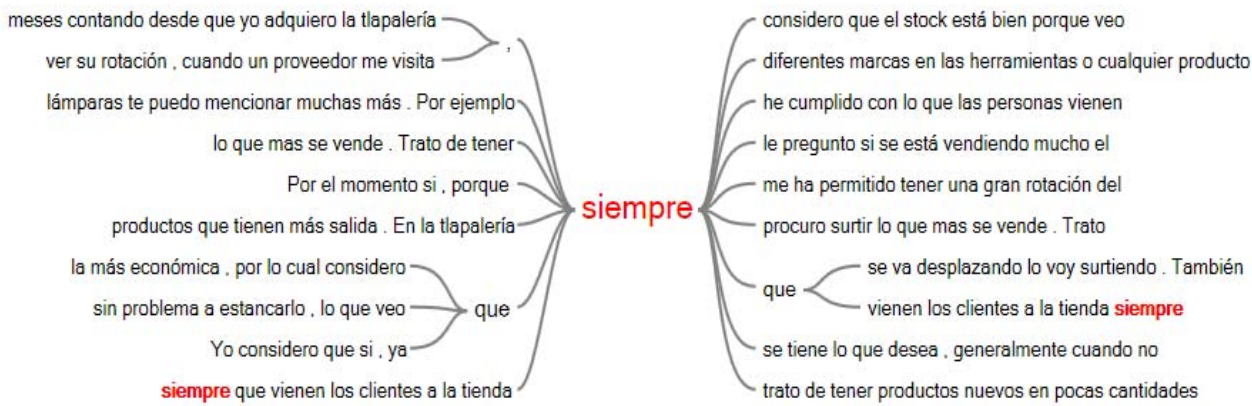

Figura 2. Red léxica de la palabra más frecuente en la herramienta enfocada al producto.

Fuente: Elaboración propia con datos arrojados por el software Nvivo 10.0.

Respecto a la herramienta enfocada al precio, la palabra con más referencias en las 4 entrevistas ante la pregunta sobre las consideraciones para establecer el precio a los productos, fue "margen"; con un total de 10 referencias y un $2.75 \%$ ponderado del total del indicado.

Observando la red léxica de la palabra "margen", se puede notar que usualmente los empleados y el propietario de la empresa la utilizan para explicar la consideración principal para establecer el precio a los productos que comercializa (ver figura 3), por ejemplo al responder "En el ramo generalmente el margen de utilidad es del 20\%, 25\%, 30\%, 40\%...", "Es como una regla no escrita es el margen de utilidad del giro..." , y 
ANÁLISIS CUALITATIVO DE LA PERCEPCIÓN DEL MARKETING COMO FACTOR COMPETITIVO INTERNO: CASO DE UNA MICROEMPRESA FERRETERA DE YUCATÁN

“...cuenta el precio de los productos y el margen de utilidad del ramo..."; de las frases anteriores también se puede afirmar que el margen de utilidad del subsector figura en las consideraciones principales para fijar precios.

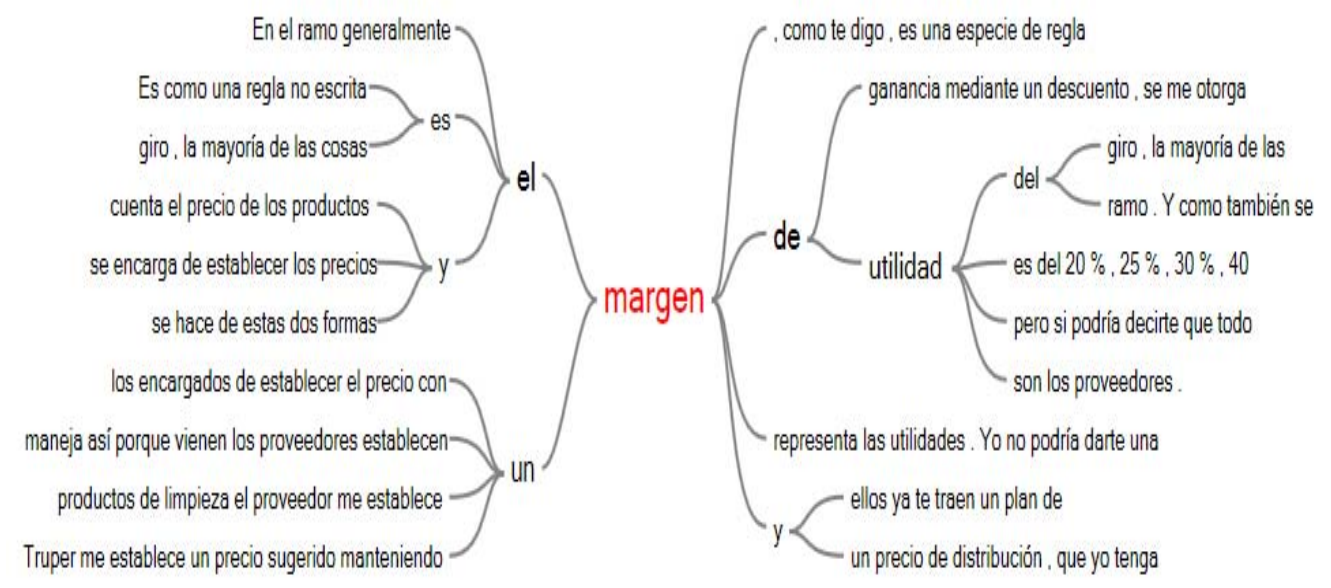

Figura 3. Red léxica de la palabra más frecuente en herramienta enfocada al precio.

Fuente: Elaboración propia con datos arrojados por el software Nvivo 10.0.

En la herramienta enfocada a la plaza, el adverbio de lugar "cerca" con 8 referencias y un total de $2.65 \%$ ponderado del total, fue la palabra más frecuente ante la pregunta sobre las consideraciones de la ubicación de la empresa, la tabla 3 muestra el total de las palabras obtenidas.

La red léxica del adverbio "cerca" se muestra en la figura 4, en ella se observa que ésta viene ligada a frases “... para nosotros es bueno tener cerca a los clientes y proveedores” ó “...las personas que más compran viven cerca o en este fraccionamiento...”; de las cuales se deduce que para la empresa la cercanía hacia los clientes y proveedores es fundamental en la ubicación de la empresa 
ANÁLISIS CUALITATIVO DE LA PERCEPCIÓN DEL MARKETING COMO FACTOR COMPETITIVO INTERNO: CASO DE UNA MICROEMPRESA FERRETERA DE YUCATÁN

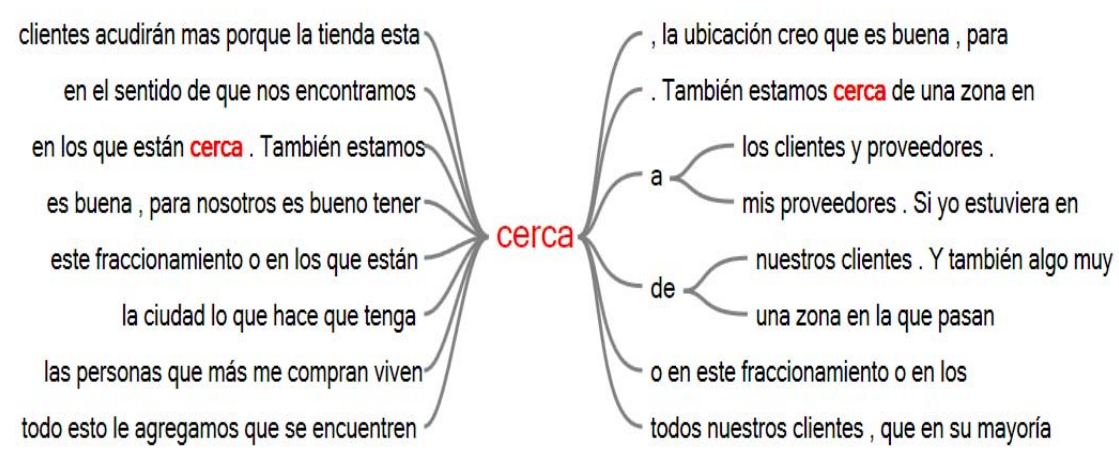

Figura 4. Red léxica de la palabra más frecuente en la herramienta enfocada a la plaza.

Fuente: Elaboración propia con datos arrojados por el software Nvivo 10.0.

En la herramienta orientada a la promoción, el análisis de frecuencia de palabras reveló que la palabra "empresa" con un total de 15 referencias y un total de $4.49 \%$ ponderado del total en las entrevistas fue la más frecuente en la pregunta sobre la importancia de la publicidad.

La red léxica de dicha palabra se muestra en la figura 5, en ella se visualizan algunas de las respuestas por ejemplo: “...es importante porque permite dar a conocer a la empresa, que la gente sepa que existe esta empresa" y "La considero importante porque ayuda a difundir la empresa y a que se den a conocer los servicios...", lo anterior demuestra que la publicidad es más importante para posicionar el nombre de la empresa y con ello ser una opción de compra en los clientes. 


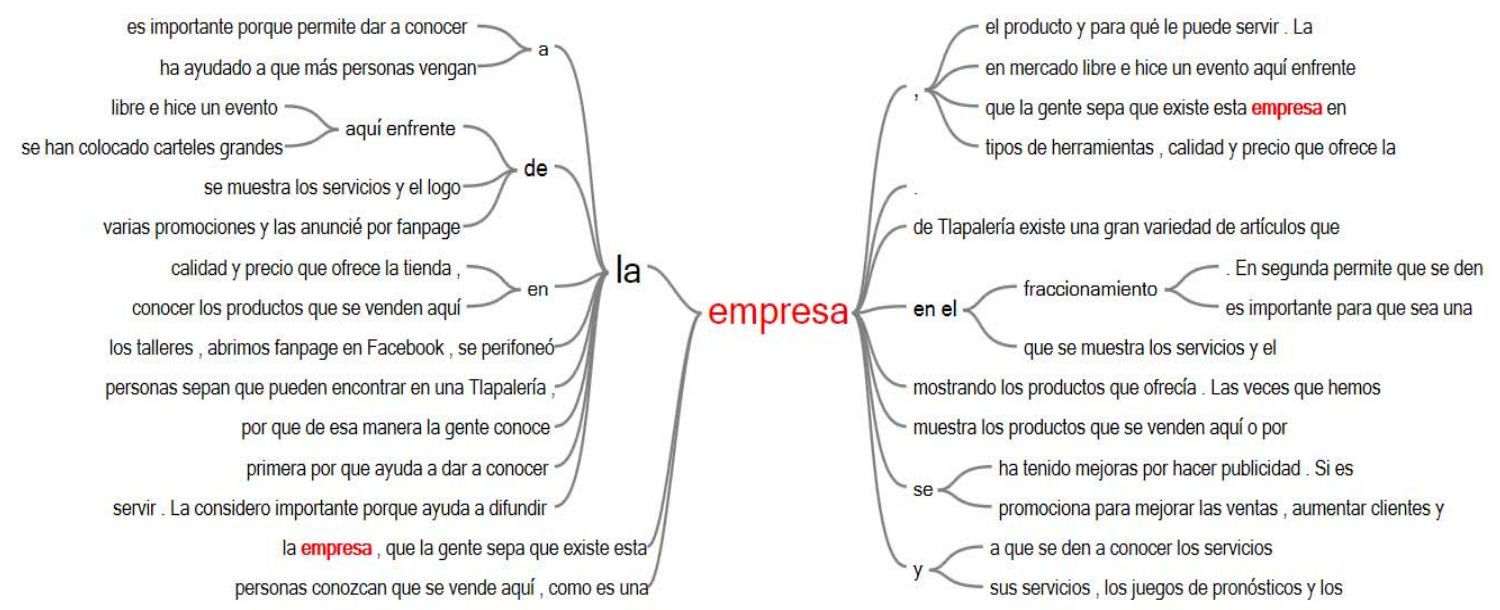

Figura 5. Red léxica de palabra más frecuente en la herramienta enfocada a la promoción.

Fuente: Elaboración propia con datos arrojados por el software Nvivo 10.0.

\section{CONCLUSIONES}

Las percepciones del propietario y de los empleados de la empresa indicaron que las actividades realizadas en el proceso de desarrollo de las herramientas operativas del marketing concuerdan con las implicaciones de cada una de dichas herramientas operativas proporcionadas por Arriaga et al. (2012).

Lo anterior, debido a que en la herramienta enfocada al producto las percepciones revelaron el compromiso de la empresa por contar con una diversidad de marcas y productos, lo cual es el principal requisito para considerar un surtido óptimo. Respecto a las consideraciones de fijación de precios a los productos, las percepciones coincidieron con lo señalado en la literatura debido a que indicaron que la empresa considera un margen mínimo de costo hacia el cliente. Las percepciones en la herramienta enfocada a la plaza revelan que la empresa considera los factores principales en la determinación de la ubicación; es decir, la cercanía con sus clientes y proveedores. Finalmente, en la herramienta publicidad las frases ligadas a la palabra más frecuente indican que las 
percepciones se inclinan a promocionar en mayor grado la empresa y en menor grado a los productos que comercializa.

Sin embargo, como se explica en la literatura, para obtener resultados óptimos del marketing, se requiere de la implementación eficiente de sus herramientas operativas lo que implica establecer un proceso formal para su ejecución (Mercado, 2004; Arriaga et al., 2012), lo cual se observó como una debilidad en la empresa especialmente en los aspectos referentes al producto, precio y plaza ya que se realizan de manera informal o empírica; en la herramienta producto las percepciones evidenciaron la ausencia de una metodología para determinar los productos a comercializar; respecto a las consideraciones fijación de precios, la empresa considera a dicha actividad como una "regla no escrita" indicando con esto informalidad; en la herramienta plaza no se efectúa un análisis que fundamente la cercanía a sus clientes y proveedores; y finalmente en la herramienta publicidad se observó divergencia en su objetivo principal el cual recae en la promoción de los productos o servicios y no en posicionar el nombre de la empresa como fue el caso de la presente investigación. 
ANÁLISIS CUALITATIVO DE LA PERCEPCIÓN DEL MARKETING COMO FACTOR COMPETITIVO INTERNO: CASO DE UNA MICROEMPRESA FERRETERA DE YUCATÁN

\section{REFERENCIAS BIBLIOGRÁFICAS}

Aguirre, M., \& Aparicio, M. (2002). La gestión de la calidad y el marketing interno como factores de competitividad en empresas de servicios: El caso de empresas vascas de servicios con gestión avanzada. Cuadernos de gestión, 2 (2), 27-50.

Arriaga, L., Avalos, A., \& De la Torre, M. (2012). Marketing mix: la fortaleza de las grandes empresas. Recuperado el 21 de 11 de 2014, de http://www.eumed.net/ce/2012/marketing-mix.html.

Bacon, M. (1996). Como hacer marketing directo: secretos para la pequeña empresa. Argentina: Granica.

Bernal, C. (2010). Metodología de la investigación. Administración, economía, humanidades y ciencias sociales. (3ra ed.). Colombia: Pearson Educación.

Camacho, M., \& Pérez, M. (2014). Aspectos que influyen en las competencias estratégicas didáctico curriculares para atender al alumnado sordo desde la voz del profesorado. Revista de curriculum y formación del profesorado , 18 (1), 341-366.

Carmona, A. (2015). Con su poder económico, The Home Depot sepulta a pequeñas ferreterías. Recuperado el 26 de Febrero de 2015, de La extra noticias: http://laextranoticias.mx/con-su-poder-economico-the-home-depot-sepulta-apequenas-ferreterias/\#.

Caro, F., \& Díez, E. (2005). Investigación cualitativa asistida por ordenador en economía de la empresa. Investigaciones Europeas de Dirección y Economía de la Empresa ,11 (2), 45-58.

Castillo, A. (2015). Expectativas del sector ferretero para 2015. Recuperado el 10 de Febrero de 2015, de Mundo ferretero: http://www.mundoferretero.com.mx/index.php/ferreteria/item/97-expectativas-delsector-ferretero-para-2015.

Castillo, V., Bojórquez, M., \& Pérez, A. (2013). La mercadotecnia, factor clave para la competitividad organizacional. Gestión y Sociedad , 6 (1), 15-30. 
ANÁLISIS CUALITATIVO DE LA PERCEPCIÓN DEL MARKETING COMO FACTOR COMPETITIVO INTERNO: CASO DE UNA MICROEMPRESA FERRETERA DE YUCATÁN

Dvoskin, R. (2004). Fundamentos de marketing: teoría y experiencia. Argentina: Ediciones Granica S.A.

Gil, E., Conti, F., Pinzon, S., Prieto, M., Solas, O., \& Cruz, M. (2002). El análisis de texto asistido por ordenador en la investigación cualitativa. Index Enferm (36), 24-28.

Hernández, R., Fernández, C., \& Baptista, P. (2010). Metodología de la Investigación (Quinta ed.). México, D.F.: Mc Graw Hill.

Home Depot invertirá 1,500 mdp en el 2016. (2015). El Economista. Recuperado el 11 de 12 de 2015, de http://eleconomista.com.mx/comment/reply/585142.

Instituto Nacional de Estadística y Geografía (INEGI). (2014). Caída de ventas en Febrero 2014. Recuperado el 20 de 04 de 2014, de http://webcache.googleusercontent.com/search?q=cache:IpZkrHP9Y4cJ:mx.m.globe dia.com/inegi-caida-ventas-febrero-2014+\&cd=4\&hl=es\&ct=clnk\&gl=mx.

Instituto Nacional de Estadística y Geografía (INEGI). (2013). Encuesta mensual de establecimientos comerciales. Recuperado el 10 de 1 de 2013 , de http://www.unionyucatan.mx/articulo/2013/10/21/economia/caen-22-anual-ventas-almenudeo-en-agosto-inegi.

Instituto Nacional de Estadística y Geografía (INEGI). (2012). Perspectiva Estadística Yucatán. Recuperado el 01 de 10 de 2014, de http://www.inegi.org.mx/prod_serv/contenidos/espanol/bvinegi/productos/integracion/ estados/persc_estd/yuc/Pers-yuc.pdf.

Kotler, P., \& Armstrong, G. (2008). Fundamentos de marketing (Octava ed.). México: Pearson Educación.

Kotler, P., \& Keller, L. (2006). Dirección de Marketing (Duodécima edición ed.). México: Pearson Educación.

López, B., Mas, M., \& Viscarri, J. (2008). Los pilares del marketing. España: Edicions UPC.

O'Shaughnessy, J. (1991). Marketing competitivo (Segunda ed.). Madrid: Díaz de Santos, S. A. 
Paredes, M. (2011). El proceso de marketing en las pequeñas empresas. Revista Académica ECO (6), 53-62.

Reyes, A. (2002). La llave china de los ferreteros. Recuperado el 28 de 07 de 2014, de http://www.imcyc.com/cyt/agosto02/llave.htm.

Rodríguez, G., Gil, J., \& García, E. (1999). Metodología de la investigación cualitativa (Segunda ed.). Málaga: Ediciones Aljibe.

Rubio, A., \& Aragón, A. (2002). Factores explicativos del éxito competitivo. Un estudio empírico en la pyme. Cuadernos de Gestión , 2 (1), 49-63.

SCIAN. (2007). Sistema de Clasificación Industrial de América del Norte. Recuperado el 30 de 09 de 2014, de http://www.inegi.org.mx/sistemas/scian/contenidos/SCIAN\%20M\%C3\%A9xico\%202 007\%20(26enero2009).pdf.

Soriano, C. (1990). Marketing mix: Conceptos, Estrategia y Aplicaciones. Madrid: Ediciones Díaz de Santos.

Stake, R. (2007). Investigación con estudio de casos (4a ed.). Madrid: Morata.

Stokes, D. (2000). Putting Entrepreneurship into Marketing: The processes of Entrepreneurial Marketing. Journal of Research in Marketing \& Entrepreneurship , 2 (1), 1-16. 